\title{
Derivation of spin-orbit couplings in collinear linear-response TDDFT: A rigorous formulation
}

Cite as: J. Chem. Phys. 140, 144103 (2014); https://doi.org/10.1063/1.4870010

Submitted: 05 February 2014 . Accepted: 19 March 2014 . Published Online: 09 April 2014

Felipe Franco de Carvalho, Basile F. E. Curchod, Thomas J. Penfold, and Ivano Tavernelli

\section{ARTICLES YOU MAY BE INTERESTED IN}

A simplified relativistic time-dependent density-functional theory formalism for the calculations of excitation energies including spin-orbit coupling effect

The Journal of Chemical Physics 123, 154102 (2005); https://doi.org/10.1063/1.2061187

Nonadiabatic dynamics with intersystem crossings: A time-dependent density functional theory implementation

The Journal of Chemical Physics 143, 224105 (2015); https://doi.org/10.1063/1.4936864

Molecular dynamics with electronic transitions

The Journal of Chemical Physics 93, 1061 (1990); https://doi.org/10.1063/1.459170

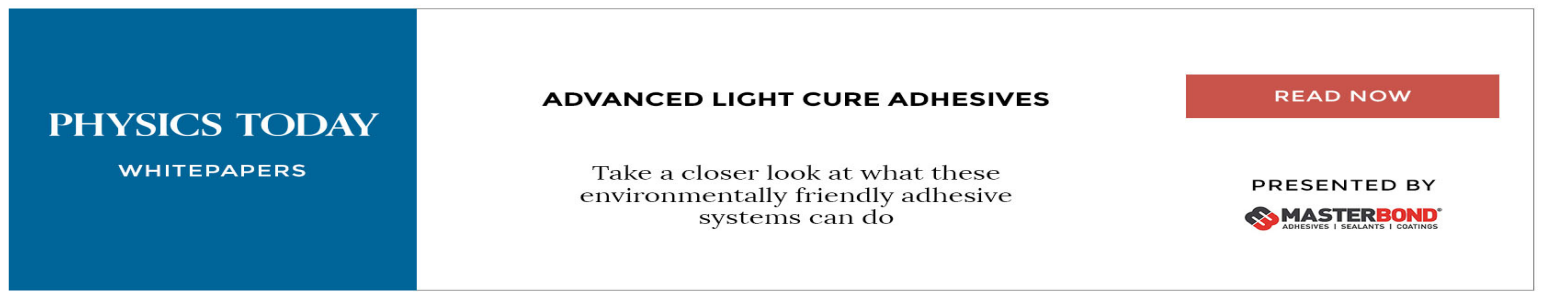




\title{
Derivation of spin-orbit couplings in collinear linear-response TDDFT: A rigorous formulation
}

\author{
Felipe Franco de Carvalho, ${ }^{1}$ Basile F. E. Curchod, ${ }^{1}$ Thomas J. Penfold, ${ }^{2}$ \\ and Ivano Tavernelli ${ }^{1, a)}$ \\ ${ }^{1}$ Laboratory of Computational Chemistry and Biochemistry, Ecole Polytechnique Fédérale de Lausanne, \\ CH-1015, Switzerland \\ ${ }^{2}$ SwissFEL, Paul Scherrer Inst, CH-5232 Villigen, Switzerland
}

(Received 5 February 2014; accepted 19 March 2014; published online 9 April 2014)

\begin{abstract}
Using an approach based upon a set of auxiliary many-electron wavefunctions we present a rigorous derivation of spin-orbit coupling (SOC) within the framework of linear-response time-dependent density functional theory (LR-TDDFT). Our method is based on a perturbative correction of the nonrelativistic collinear TDDFT equations using a Breit-Pauli spin-orbit Hamiltonian. The derivation, which is performed within both the Casida and Sternheimer formulations of LR-TDDFT, is valid for any basis set. The requirement of spin noncollinearity for the treatment of spin-flip transitions is also discussed and a possible alternative solution for the description of these transitions in the collinear case is also proposed. Our results are validated by computing the SOC matrix elements between singlet and triplet states of two molecules, formaldehyde and acetone. In both cases, we find excellent agreement with benchmark calculations performed with a high level correlated wavefunction method. (C) 2014 AIP Publishing LLC. [http://dx.doi.org/10.1063/1.4870010]
\end{abstract}

\section{INTRODUCTION}

The relativistic equivalent of the time-dependent Schrödinger equation involves the solution of the Dirac equation and its many-electron extensions. Relativistic quantum chemistry is an active area of research and there have been several recent reviews on the subject. ${ }^{1-3}$ Even though generally small, relativistic corrections can have an important influence on the energy levels, orbital shapes, and geometries of molecules. ${ }^{4}$

An alternative to fully relativistic calculations is to add relativistic corrections to the standard, non-relativistic electronic structure theories. The best known corrections are the mass-velocity, Darwin, and spin-orbit coupling (SOC) terms. The first two are usually called scalar relativistic effects because they do not involve vector operators. On the other hand, spin-orbit coupling acts on both angular momentum and spin, leading to its defining characteristic of mixing orbital and spin degrees of freedom, thus allowing electronic states of different multiplicities to couple. Consequently, SOCs have a wide range of important effects in chemistry and physics, such as fine-structure and band splitting in molecules, semiconductors, and metals, molecular magnetism, spin transport in spintronics and magnetoelectronics, spin quantum dots, and qubits dynamics. In particular, SOC can also be very important for photochemistry because it turns spin-forbidden processes, such as intersystem crossing and phosphorescence into weakly allowed transitions.

In this respect, SOCs are also particularly important for molecular dynamics because they allow for intersystem crossings (ISC) between electronic states of different spin multiplicity. The description of these processes requires the

\footnotetext{
a)Electronic mail: ivano.tavernelli@epfl.ch
}

evaluation of SOC matrix elements between different electronic states, and thus involves the use of excited states methods such as configuration interaction singles (CIS) or linear-response time-dependent density functional theory (LR-TDDFT). In particular, the use of Kohn-Sham DFT ${ }^{5}$ and $\mathrm{TDDFT}^{6}$ approaches is particularly well suited for the calculation of SOC in large molecular systems where a good balance between numerical efficiency and accuracy is required.

Within DFT/LR-TDDFT two main routes for the calculation of relativistic spin-orbit effects can be explored, which differ in the way the density functionalization of the relativistic Schrödinger equations is performed. When the Dirac equations are directly worked into the Kohn-Sham formalism one obtains the single-particle equations of relativistic DFT (KS-RDFT), which have the form of the Dirac equation with a current-dependent one-particle four-potential $v_{s}^{\mu}(\mathbf{r}) .^{7,8}$ This approach is the starting point for approximations, such as the quasi-relativistic "Zeroth Order Regular Approximation" (ZORA) Hamiltonian as implemented within the Amsterdam Density Functional (ADF) program. ${ }^{9}$ In this case, a two-component calculation is performed, and all relativistic effects are included variationally to the one-component non-relativistic calculation. As a consequence, relativistic corrections also affect the electronic density and the wavefunction, instead of just the energy as in the case of the perturbative treatment. However, such two-component calculations are very computationally demanding and for this reason approximate solutions to these equations have also been proposed. ${ }^{10}$

Alternatively, one can compute the SOC perturbatively starting from the one-component matrix element of the BreitPauli Hamiltonian in its many-body formulation, and perform the density functionalization of the first-order relativistic corrections to the non-relativistic energies. The major challenge 
of this approach within TDDFT is that the matrix elements of the SOC Hamiltonian are not a simple functional of the electronic density (by virtue of their definition involving manyelectron wavefunctions). To overcome this difficulty, we apply a method based on the so-called "auxiliary" set of many-electron wavefunctions (named AMEW) derived from LR-TDDFT quantities, which enables the calculation of in principle exact LR-TDDFT matrix elements of any given onebody operator. ${ }^{1-13}$ This formalism has already been successfully applied to the calculation of nonadiabatic coupling vectors (NACVs). ${ }^{11,13,14}$

In this work, we present a derivation of SOC matrix elements within the AMEW formalism in context of LR-TDDFT and describe its implementation into the CPMD program. ${ }^{15}$ A similar approach to calculate SOCs in molecules based on Casida's 16 "singly excited configurations" has already been proposed by Chiodo and Russo ${ }^{17}$ and recently by Subotnik and co-workers, ${ }^{18}$ but these were done in an ad hoc fashion, following the analogies with wavefunction theory techniques such as CIS. Here, we base our implementation on the rigorous results presented in Refs. 11, 13, and 14, and investigate the use of conventional collinear DFT functionals and their functional derivatives in the calculation of collinear and noncollinear (spin-flip) coupling terms. Our formalism can also be easily extended, using the development in Ref. 14, to the calculation of SOC between pairs of excited states, which is of paramount importance for the evaluation of ISC events in nonadiabatic molecular dynamics calculations. In addition, in this paper we derive the working equation for the calculation of the SOC matrix elements within the so-called Casida ${ }^{16}$ and Sternheimer ${ }^{19,20}$ formulations of LR-TDDFT, which can be applied to localized as well as plane wave basis set calculations.

Finally, we would like to stress that the implementation of this efficient method for the evaluation of the SOC in any DFT/LR-TDDFT-based molecular dynamics package (together with the calculation of NACVs ${ }^{11-13}$ ) will allow the "on-the-fly" calculation of both kinds of nonradiative energy transfer phenomena-internal conversion and intersystem crossing - for large systems (isolated and in condensed phase) at a good level of accuracy. Similar studies based on different electronic structure approaches have already appeared in the literature..$^{21,22}$

\section{THEORY}

The relativistic corrections to the conventional KohnSham DFT equations were originally derived by Rajagopal and Callaway from quantum electrodynamics. ${ }^{23}$ In the noncollinear case the exchange and correlation functional can be given as a functional of the electron density $\rho$ and of the spin magnetization density ${ }^{24}$

$$
\mathbf{m}(\mathbf{r})=\mu_{B} \sum_{i, \nu_{1}, \nu_{2}} \psi_{i}^{\dagger}\left(\mathbf{r}, v_{1}\right) \bar{\sigma}_{\nu_{1}, \nu_{2}} \psi_{i}\left(\mathbf{r}, v_{2}\right),
$$

where $v_{i} \in\{\alpha, \beta\}, \mu_{B}=\hbar q / 2 m$ is the Bohr magneton, $\psi_{i}\left(\mathbf{r}, v_{1}\right)$ is an element of the two-component KS-spinors

$$
\boldsymbol{\psi}_{i}(\mathbf{r})=\left(\begin{array}{l}
\psi_{i}(\mathbf{r}, \alpha) \\
\psi_{i}(\mathbf{r}, \beta)
\end{array}\right)
$$

whose spin-axes are not necessarily aligned along the main spin quantization axis (z-axis). $\bar{\sigma}=\left(\sigma_{1}, \sigma_{2}, \sigma_{3}\right)$ is the vector of Pauli matrices. ${ }^{25,26}$

In the collinear local spin density approximation (LSDA), ${ }^{27}$ each electronic spin is oriented along a fixed quantization axis ( $z$-axis) and the spinors can therefore be written in the spin-up $(\alpha)$ and spin-down $(\beta)$ forms

$$
\boldsymbol{\psi}_{i, \alpha}(\mathbf{r})=\left(\begin{array}{c}
\psi_{i}(\mathbf{r}, \alpha) \\
0
\end{array}\right), \quad \boldsymbol{\psi}_{i, \beta}(\mathbf{r})=\left(\begin{array}{c}
0 \\
\psi_{i}(\mathbf{r}, \beta)
\end{array}\right)
$$

or in the direct product space spanned by the Hilbert space of the KS-orbitals, $H_{K S}$ and the two-dimensional spin space $H_{\text {Spin }}$, as

$$
\boldsymbol{\psi}_{i, \alpha}(\mathbf{r})=\psi_{i}(\mathbf{r}) \otimes\left(\begin{array}{l}
1 \\
0
\end{array}\right), \quad \boldsymbol{\psi}_{i, \beta}(\mathbf{r})=\psi_{i}(\mathbf{r}) \otimes\left(\begin{array}{l}
0 \\
1
\end{array}\right) .
$$

The spin magnetization then becomes

$$
m_{z}(\mathbf{r})=\mu_{B}\left[\sum_{i}^{N_{\alpha}}\left|\psi_{i, \alpha}(\mathbf{r})\right|^{2}-\sum_{i}^{N_{\beta}}\left|\psi_{i, \beta}(\mathbf{r})\right|^{2}\right],
$$

where $N_{\alpha}$ and $N_{\beta}$ are the number of occupied $\alpha$ and $\beta$ KS orbitals, respectively, and $m_{x}(\mathbf{r})=m_{y}(\mathbf{r})=0$. A timedependent spin DFT for the corresponding dynamic spin susceptibility was derived by Vosko and co-workers. ${ }^{28}$

In this work, we do not derive a relativistic version of the LR-TDDFT equations, which would include the SOC terms explicitly in the reference relativistic ground state KS Hamiltonian. Instead, starting from nonrelativistic KS-DFT and (standard) collinear LR-TDDFT equations we compute, perturbatively, the relativistic effects associated with the SOC Hamiltonian in the Breit-Pauli approximation, $\hat{H}_{B P}^{S O}$. This is achieved by a density functionalization of the matrix elements of $\hat{H}_{B P}^{S O}$ using a LR-TDDFT-based reconstruction of the many-electron wavefunctions of the ground and excited states according to the formalism derived in Refs. 11-13. Our approach is general and can be applied to any type of approximate SOC Hamiltonian in the perturbative approach, including the two-component ZORA Hamiltonian. ${ }^{9}$

A noncollinear formulation of TDDFT was developed by Shao et al. ${ }^{29}$ and Wang and Ziegler ${ }^{30,31}$ for two-component spinors (see also the book of Ullrich ${ }^{32}$ ). Unlike conventional (collinear) LR-TDDFT, noncollinear TDDFT can describe electronic transitions that involve spin-flips. These are of importance when evaluating SOC between states with different spin quantum number $M_{S}$ as in the case of a singlet to triplet transition between states $\frac{1}{\sqrt{2}}(|\alpha \beta\rangle-|\beta \alpha\rangle)\left(M_{S}=0\right)$ and $|\alpha \alpha\rangle$ $\left(M_{S}=1\right)$, for a two-electron system. Despite recent progress in this field, very little has been done concerning the application of noncollinear TDDFT to molecular systems beyond the local-density approximation (LDA) of the DFT functional and the TDDFT kernel. Here, we only consider the collinear form of LR-TDDFT, from which we can derive without further approximations the SOC terms between singlet and triplet states that share the same $M_{s}$ value (i.e., those excitations that can be reached from the ground state without spin-flip). The approximate extension of this theory to the spin-flip case will be presented and discussed in Sec. II A. 
In full, the Breit-Pauli SOC operator is composed of two parts: a one-electron term (which involves spin-same-orbit coupling only) and a two-electron term (which involves spinsame-orbit and spin-other-orbit coupling). Briefly, the oneelectron term describes the interaction between the magnetic spin moment, $\boldsymbol{\mu}_{e l}^{S}=-g_{S} \mu_{B} \boldsymbol{S} / \hbar$, of an electron with the magnetic moment, $\boldsymbol{\mu}_{e l}^{L}=-g \mu_{B} \boldsymbol{L} / \hbar$, induced by its orbiting in the nuclear electrostatic field (where $\mu_{B}$ is the Bohr magneton, and $g_{S}$ and $g$ are dimensionless factors). Analogously, when the coupling occurs in the electric field generated by another electron we obtain the two-electron (spin-same-orbit) coupling term. Finally, the two-electron spin-other-orbit terms arise from the interaction of the spin magnetic moment of one electron with the orbital magnetic moment of a second one, and vice versa. These two terms provide screening of the one-electron term similarly to the contributions of the nuclear-electron attraction and electron-electron repulsion interactions in the Born-Oppenheimer (BO) Hamiltonian (see Appendix A). Importantly, while for a full quantitative description of the SOC matrix elements, both the one and two matrix elements are required, the one electron contributions increase much faster with nuclear charge ${ }^{2}$ than the two electron terms and therefore for many cases, as used here, the oneelectron term is dominant.

The one-electron term of the Breit-Pauli Hamiltonian is expressed as

$$
\hat{H}_{1 e l}^{S O}=\frac{\alpha^{2}}{2}\left[\sum_{\gamma=1}^{N_{\gamma}} \sum_{i=1}^{N} Z_{\gamma} \frac{1}{\hat{r}_{i \gamma}^{3}}\left(\hat{\mathbf{l}}_{i \gamma} \cdot \hat{\mathbf{s}}_{i}\right)\right]
$$

and it can be evaluated in a formally exact way within LRTDDFT using the AMEW method introduced in Refs. 13 and 33. In Eq. (6) $\alpha=\frac{e^{2}}{4 \pi \epsilon_{0} \hbar c}$ is the fine structure constant, the first sum runs over all nuclei and the second one over all electrons, $Z_{\gamma}$ is the charge of nucleus $\gamma, r_{i \gamma}$ is the distance between nucleus $\gamma$ and electron $i, \hat{\mathbf{i}}_{i \gamma}$ is the angular momentum of electron $i$ with respect to nucleus $\gamma$, and $\hat{\mathbf{s}}_{i}$ is the spin operator of electron $i$ with components $s_{i}=(\hbar / 2) \sigma_{i}(i=x, y, z)$. We will use atomic units throughout, i.e., $\alpha=c^{-1}$ and $\hbar=1$.

\section{A. The LR-TDDFT equations}

\section{Casida's formalism}

In the adiabatic approximation, ${ }^{16,34}$ the LR-TDDFT equations can be formulated in the well known Casida's form

$$
\Omega \mathbf{F}^{I}=\omega_{I}^{2} \mathbf{F}^{I}
$$

with

$$
\Omega=(A-B)^{1 / 2}(A+B)(A-B)^{1 / 2}
$$

and

$$
\begin{gathered}
A_{i a \xi, j b \tau}=\delta_{\xi, \tau} \delta_{i, j} \delta_{a, b}\left(\epsilon_{b \tau}-\epsilon_{j \tau}\right)+K_{i a \xi, j b \tau}, \\
B_{i a \xi, j b \tau}=K_{i a \xi, b j \tau},
\end{gathered}
$$

$$
\begin{aligned}
& K_{i a \xi, j b \tau} \\
& =\iint \mathrm{d}^{3} \mathbf{r} \mathrm{r}^{3} \mathbf{r}^{\prime} \varphi_{i \xi}^{*}(\mathbf{r}) \varphi_{a \xi}(\mathbf{r}) \frac{1}{\left|\mathbf{r}-\mathbf{r}^{\prime}\right|} \varphi_{j \tau}\left(\mathbf{r}^{\prime}\right) \varphi_{b \tau}^{*}\left(\mathbf{r}^{\prime}\right) \\
& \quad+\iint \mathrm{d}^{3} \mathbf{r d}^{3} \mathbf{r}^{\prime} \varphi_{i \xi}^{*}(\mathbf{r}) \varphi_{a \xi}(\mathbf{r}) \frac{\delta^{2} E_{x c}}{\delta \rho_{\xi}(\mathbf{r}) \delta \rho_{\tau}\left(\mathbf{r}^{\prime}\right)} \varphi_{j \tau}\left(\mathbf{r}^{\prime}\right) \varphi_{b \tau}^{*}\left(\mathbf{r}^{\prime}\right) .
\end{aligned}
$$

In Eq. (8), $\omega_{I}$ are the excitation energies and $\mathbf{F}^{I}$ the corresponding eigenvectors. ${ }^{35}$ Here and in what follows, we use indices $i, j$, and $k$ for occupied KS orbitals; indices $a, b$, and $c$ for virtual KS orbitals; $p, q, r$ for general KS orbitals; and $\xi$ and $\tau$ for the spin indices. The $\varphi_{p \xi}(\mathbf{r})$ are KS orbitals associated to the KS energies $\epsilon_{p \xi}$ and the total spin density is given by $\rho_{\xi}(\mathbf{r})=\sum_{i}\left|\varphi_{i \xi}(\mathbf{r})\right|^{2}$. Notice that Eqs. (9)-(11) are written for a general case in which spin-polarization may be present, even though we will only work with closed-shell molecules which means that $\rho_{\alpha}(\mathbf{r})=\rho_{\beta}(\mathbf{r})$.

Recently, in a series of papers ${ }^{11-13}$ we demonstrated how the eigenvectors $\mathbf{F}^{I}$ of Eq. (7) can be used to construct a manyelectron wavefunction ansatz $\left|\Psi_{I}\right\rangle$ for electronic state $I$ named AMEW

$$
\left|\Psi_{I}\right\rangle=\sum_{i}^{N} \sum_{a}^{\infty} \sum_{\xi=\{\alpha, \beta\}} c_{i a \xi}^{I} \hat{a}_{a \xi}^{\dagger} \hat{a}_{i \xi}\left|\Psi_{0}\right\rangle,
$$

where $\left|\Psi_{0}\right\rangle$ is the Slater determinant (SD) of all occupied KS orbitals, $\hat{a}_{a \xi}^{\dagger}$ and $\hat{a}_{i \xi}$ are the KS creation and annihilation operators, respectively, and $c_{i a \xi}^{I}=\left(\left(\epsilon_{a \xi}-\epsilon_{i \xi}\right) / \omega_{I}\right)^{1 / 2} F_{i a \xi}^{I}$. The quantities $F_{i a \xi}^{I}$ are the components of the eigenvector $\mathbf{F}^{I}$ of Casida's equation associated to the excitation energy $\omega_{I}$. The sum over $a$ has to be truncated in practice when a finite number of virtual orbitals is used in the calculation. Equation (12) describes the AMEW as a linear combination of singly excited SDs. It is important to stress the fact that this is not the actual many-electron wavefunction of state $I$, however it has been shown that $\left|\Psi_{I}\right\rangle$ in Eq. (12) provides the exact matrix elements of one-electron operator $\hat{O}$ computed for the ground state and any excited state, $\left\langle\Psi_{0}|\hat{O}| \Psi_{I}\right\rangle \cdot{ }^{13}$ It has also been shown that matrix elements of $\hat{O}$ between pairs of excited states $I$ and $J,\left\langle\Psi_{I}|\hat{O}| \Psi_{J}\right\rangle$ can be calculated approximately using the AMEW. ${ }^{14}$

\section{The Sternheimer formalism}

An alternative approach to Casida's equations is the Sternheimer formalism for DFT/LR-TDDFT. ${ }^{19,20,36-38}$ In this case only ground-state (occupied) KS orbitals are used $\left\{\varphi_{k \xi}\right\}$ together with their corresponding linear-response perturbed orbitals $\left\{\phi_{k \xi}\right\}$. The advantage of this formalism is that it requires only $N$ occupied and $N$ LR orbitals (while in the Casida's approach it is not always obvious how many virtual orbitals should be included a priori in the calculation).

The Sternheimer approach is based on the first-order time-dependent perturbative expansion of the unperturbed KS orbitals

$$
\varphi_{k \xi}^{\prime}(\mathbf{r}, t)=\varphi_{k \xi}(\mathbf{r})+\lambda \phi_{k \xi}(\mathbf{r}, t)
$$


where $\lambda$ is a small coupling parameter that is only used to keep track of the order of the perturbation in the derivation of the Sternheimer equations. Then the time-dependent perturbed density can be written as

$$
\begin{aligned}
\rho(\mathbf{r}, t)= & \sum_{i}^{N} \sum_{\xi}\left|\varphi_{i \xi}(\mathbf{r})\right|^{2} \\
& +\lambda \sum_{i}^{N} \sum_{\xi}\left[\phi_{i \xi}^{*}(\mathbf{r}, t) \varphi_{i \xi}(\mathbf{r})+\varphi_{i \xi}^{*}(\mathbf{r}) \phi_{i \xi}(\mathbf{r}, t)\right] \\
= & \rho^{(0)}(\mathbf{r})+\lambda \rho^{(1)}(\mathbf{r}, t) .
\end{aligned}
$$

Inserting Eq. (14) into the TD-KS equations and equating the coefficients with equal powers of $\lambda$ we obtain a set of coupled equations for $\phi_{k \xi}(\mathbf{r}, t)$ and $\varphi_{k \xi}(\mathbf{r})$, with the orthogonality condition $\left\langle\varphi_{i} \mid \phi_{j}\right\rangle=0, \forall i, j$ and $\forall t$. Assuming the adiabatic approximation and considering a harmonic perturbation of the type $\delta V(\mathbf{r}, t)=\delta V(\mathbf{r}) e^{i \omega t}$, we finally arrive at the following set of equations for the LR KS orbitals $\phi_{k}^{I}(\mathbf{r}, t)$,

$$
\left[\epsilon_{k}-\omega_{I}-H^{K S}\right] \phi_{k}^{I}(\mathbf{r}, \omega)=\mathcal{Q}\left[\delta V(\mathbf{r})+\delta V^{S C F}(\omega)\right] \varphi_{k}(\mathbf{r}, \omega),
$$

in the frequency domain, where $\mathcal{Q}=1-\sum_{i=1}^{N}\left|\varphi_{i}\right\rangle\left\langle\varphi_{i}\right|$ in Eq. (15) is the projector onto the subspace of virtual KS orbitals, and $I$ labels the different solutions (that correspond to the different excited states). By expanding both the GS KS orbitals and the LR orbitals in a finite basis set, Eq. (15) can be recast into an eigenvalue problem (in the adiabatic approximation) similar to Casida's equations. ${ }^{20}$

In this formalism, the AMEW expansion becomes ${ }^{14}$

$$
\left|\Psi_{I}\right\rangle=\sum_{i}^{N} \sum_{\xi}\left(\hat{r}_{i \xi}^{I}\right)^{\dagger} \hat{a}_{i \xi}\left|\Psi_{0}\right\rangle
$$

where $\left(\hat{r}_{i \xi}^{I}\right)^{\dagger}$ is the creation operator for the LR orbitals, which can be expressed as $\left(\hat{r}_{i \xi}^{I}\right)^{\dagger}=\sum_{a}^{N} c_{i a \xi}^{I} \hat{a}_{a \xi}^{\dagger}$, and therefore $\phi_{i \xi}^{I}(\mathbf{r})=\sum_{a} c_{i a \xi}^{I} \varphi_{a \xi}(\mathbf{r})$ with $c_{i a \xi}^{I}=\left\langle\varphi_{a \xi} \mid \phi_{i \xi}\right\rangle$, using the convention that $i, j, \ldots$ label occupied, $a, b, \ldots$ virtual, and $p, q$, $r, \ldots$ general $\mathrm{KS}$ orbitals.

\section{B. Computation of SOC using the AMEW}

\section{Multiplicity of the LR-TDDFT solutions}

As shown in Appendix B and in Ref. 16, the solution to the collinear case of Casida's equations for closed shell molecules and corresponding AMEWs can be associated to spin-adapted configurations (eigenvectors of the $S^{2}$ operator) with $M_{S}=0$ and triplet configurations.

This result can be easily extended to the general case of an $N$-electron system; when $c_{i a \alpha}^{I}=c_{i a \beta}^{I}$ the corresponding AMEW describes a singlet, while if $c_{i a \alpha}^{I}=-c_{i a \beta}^{I}$ the AMEW represents a triplet state with $M_{S}=0$ (see Appendix B).

In the Sternheimer representation, the excited state singlet and triplet AMEW will be given by

$$
\left|S_{I}^{0}\right\rangle=\sum_{i}^{N} \sum_{\xi}\left(\hat{r}_{i \xi}^{S_{I}}\right)^{\dagger} \hat{a}_{i \xi}\left|\Psi_{0}\right\rangle
$$

and

$$
\left|T_{I}^{0}\right\rangle=\sum_{i}^{N}\left(\hat{r}_{i \alpha}^{T_{I}}\right)^{\dagger} \hat{a}_{i \alpha}\left|\Psi_{0}\right\rangle-\left(\hat{r}_{i \beta}^{T_{I}}\right)^{\dagger} \hat{a}_{i \beta}\left|\Psi_{0}\right\rangle,
$$

respectively. In the definition of the states, the upper index gives the values of $M_{s}$, while the lower one labels the state number. In Eqs. (17) and (18), the creation operators $\left(\hat{r}_{i \xi}^{S_{I}}\right)^{\dagger}$ and $\left(\hat{r}_{i \xi}^{T_{I}}\right)^{\dagger}$ refer to the creation of LR-KS orbitals for the singlet and triplet calculations, respectively. In general, these two sets of LR orbitals are different. The spin of a given orbital is then assigned by multiplying it with the correct spin function to obtain the corresponding spin-orbital as in Eq. (4), i.e., $\varphi_{i}^{T}(\mathbf{r})=\varphi_{i}^{T}(\mathbf{r}) \alpha(\mathbf{s})$ and $\bar{\varphi}_{i}^{T}(\mathbf{r})=\varphi_{i}^{T}(\mathbf{r}) \beta(\mathbf{s})$ (spin-restricted formalism is assumed).

\section{The "spin-flip" solution}

In conventional (collinear) LR-TDDFT, only the $M_{S}=0$ component of each triplet state can be obtained, as $M_{S}=-1$ or $M_{S}=+1$ states require spin-flip excitations to be taken into account. This means that only excitations that conserve the projection of spin angular momentum are allowed. A rigorous description of the spin-flip configuration would require the noncollinear LR-TDDFT formalism introduced by Ziegler and co-workers in which electrons are described by spinor wavefunctions with different spin orientations. ${ }^{30,31}$

However, in this work we explore a simpler solution which uses the linear-response orbitals (or coefficients in the case of Casida's formalism) obtained from a non-spin-flip triplet state calculation to build the other two triplet configurations. In other words, we represent the triplets $M_{S}=1$ and $M_{S}=-1$ by

$$
\left|T_{I}^{+1}\right\rangle=\sum_{i}^{N}\left(\hat{r}_{i \alpha}^{T_{I}}\right)^{\dagger} \hat{a}_{i \beta}\left|\Psi_{0}\right\rangle
$$

and

$$
\left|T_{I}^{-1}\right\rangle=\sum_{i}^{N}\left(\hat{r}_{i \beta}^{T_{I}}\right)^{\dagger} \hat{a}_{i \alpha}\left|\Psi_{0}\right\rangle,
$$

respectively. This approach is clearly approximate, however we will leave it without a more formal justification. In Sec. IV we shall show the level of accuracy of this simple procedure by means of an application.

\section{Evaluation of the SOC matrix elements}

We now outline our strategy for the computation of the SOC matrix elements between a singlet and a triplet excited state $\left\langle S_{I}^{0}\left|\hat{H}_{1 e l}^{S O}\right| T_{J}^{M_{S}}\right\rangle$. As an example, for a system made of four electrons the matrix element $\left\langle S_{I}^{0}\left|\hat{H}_{1 e l}^{S O}\right| T_{J}^{0}\right\rangle$ will be given by the sum of 16 matrix elements involving different AMEW and the ground state SD, while in the case of $\left\langle S_{I}^{0}\left|\hat{H}_{1 e l}^{S O}\right| T_{J}^{-1,+1}\right\rangle$ this number will reduce to eight.

a. Casida approach. In this case we have an orthonormal basis of occupied and virtual KS orbitals, and therefore the 
Slater-Condon rules ${ }^{39}$ can be used to compute all of the necessary matrix elements involving AMEWs as in Eq. (12).

Within this framework the SOC matrix elements become

$$
\begin{aligned}
\left\langle\Psi_{S}\left|\hat{H}_{1 e l}^{S O}\right| \Psi_{T}\right\rangle= & \sum_{i j \xi} \sum_{a b \xi^{\prime}}\left(c_{i a \xi}^{S}\right)^{*} c_{j b \xi^{\prime}}^{T}\left\langle\Psi_{0}\right|\left(\hat{a}_{i \xi}^{S}\right)^{\dagger} \hat{a}_{a \xi}^{S} \hat{H}^{S O} \\
& \times\left(\hat{a}_{b \xi^{\prime}}^{T}\right)^{\dagger} \hat{a}_{j \xi^{\prime}}^{T}\left|\Psi_{0}\right\rangle
\end{aligned}
$$

where $i, j$ run from 1 to $N, a, b$ from 1 to (in principle) $\infty$, and $\xi, \xi^{\prime} \in\{\alpha, \beta\}$. As shown in Ref. 14, this type of matrix elements can be evaluated using reconstructed AMEWs.

b. Sternheimer approach. In the Sternheimer formalism the set of linear response orbitals for the singlet state $\left\{\varphi_{r}^{S}(\mathbf{r})\right\}$ are not orthogonal to those of the triplet states $\left\{\varphi_{r}^{T}(\mathbf{r})\right\}$ and therefore, in contrast to the Casida's formalism, we cannot use the Slater-Condon rules for calculating matrix elements of SDs. Instead, we require the more general Löwdin's rule, ${ }^{40}$

$$
\begin{aligned}
& \left\langle\Psi_{S}\left|\sum_{i=1}^{N} \bar{h}^{S O}(i)\right| \Psi_{T}\right\rangle \\
& =\left(D_{S S} D_{T T}\right)^{-1 / 2} \sum_{i, j}^{N}\left\langle\psi_{i}^{S}\left|\bar{h}^{S O}\right| \psi_{j}^{T}\right\rangle D_{S T}\left(\psi_{i}^{S}, \psi_{j}^{T}\right),
\end{aligned}
$$

where $\psi_{i}^{S}(\mathbf{r})$ are the orbitals that generate $\left|\Psi_{S}\right\rangle$ and $\psi_{j}^{T}(\mathbf{r})$ those generating $\left|\Psi_{T}\right\rangle, D_{S T}$ is the determinant of matrix $S_{S T}$ containing the overlaps between all of the spin-orbitals,

$$
S_{S T}=\left(\begin{array}{cccc}
\left\langle\psi_{1}^{S} \mid \psi_{1}^{T}\right\rangle & \left\langle\psi_{1}^{S} \mid \psi_{2}^{T}\right\rangle & \cdots & \left\langle\psi_{1}^{S} \mid \psi_{N}^{T}\right\rangle \\
\left\langle\psi_{2}^{S} \mid \psi_{1}^{T}\right\rangle & \left\langle\psi_{2}^{S} \mid \psi_{2}^{T}\right\rangle & \ldots & \left\langle\psi_{2}^{S} \mid \psi_{N}^{T}\right\rangle \\
\vdots & \vdots & \ddots & \vdots \\
\left\langle\psi_{N}^{S} \mid \psi_{1}^{T}\right\rangle & \left\langle\psi_{N}^{S} \mid \psi_{2}^{T}\right\rangle & \cdots & \left\langle\psi_{N}^{S} \mid \psi_{N}^{T}\right\rangle
\end{array}\right),
$$

and $D_{S T}\left(\psi_{i}^{S}, \psi_{j}^{T}\right)$ is the cofactor of element $[i, j]$ (i.e., $(-1)^{i+j}$ times the determinant obtained by removing the $i$ th row and $j$ th column of $S_{S T}$ ). Notice that in our case $D_{S S}$ $=D_{T T}=1$. In Eq. (22), $\bar{h}^{S O}$ refers to the 3 dimensional vector $\frac{\alpha^{2}}{2}\left[\sum_{\gamma=1}^{N_{\gamma}} \sum_{i=1}^{N} Z_{\gamma}\left(1 / r_{i \gamma}^{3}\right) \mathbf{l}_{i \gamma}\right]$ (see Eq. (6)). More detailed information on the evaluation of Eq. (22) is given in Appendix C.

The computation of the two-electron term within the auxiliary many-electron wavefunction approach to LR-TDDFT will require further theoretical investigation, which is clearly beyond the scope of this work. However, this term is in general of opposite sign compared to the one-electron contribution and its effect is commonly accounted for through a rescaling of the atomic number $Z$ of each element to a (smaller) effective value $Z_{\text {eff. }}{ }^{41}$ The one-electron term is especially dominant for heavier atoms because it scales as $\sim Z^{2}$. $^{2}$

\section{NUMERICAL IMPLEMENTATION}

In the discussion about Eq. (6) we showed that for the evaluation of the one-electron SOC matrix elements we only need to compute one-electron integrals of the form

$$
\left\langle\left.\psi_{r}\left|\left(\hat{\mathbf{r}}_{i \gamma} \times \hat{\mathbf{p}}_{i}\right) /\right| \mathbf{r}_{i \gamma}\right|^{3} \mid \psi_{s}\right\rangle \cdot\left\langle\xi_{r}|\hat{\mathbf{s}}| \xi_{s}\right\rangle
$$

where $\psi_{i}$ represents a generic KS or LR-KS orbital, $\left\{\hat{\mathbf{r}}_{i}, \hat{\mathbf{p}}_{i}\right\}$ are the electron position and momentum, $\mathbf{r}_{i \gamma}=\mathbf{r}_{i}-\mathbf{R}_{\gamma}, \mathbf{R}_{\gamma}$ is a nuclear position, and $\xi_{r}, \xi_{s} \in\{\alpha, \beta\}$. The spin part of this matrix element is easy to compute. If we choose our quantization axis to be $z$, then the one-electron spin operator $\mathbf{s}_{i}$ is a vector of Pauli matrices $\sigma_{i}$, which act on the spin states $|\alpha\rangle$ $=\left(\begin{array}{ll}1 & 0\end{array}\right)^{T}$ and $|\beta\rangle=\left(\begin{array}{ll}0 & 1\end{array}\right)^{T}$.

The calculation of the first factor in Eq. (24) is numerically more involved since it requires the evaluation of integrals of the form $\left\langle\psi_{1}\left|\frac{\hat{r}_{y} \hat{p}_{z}}{r_{\gamma i}^{3}}\right| \psi_{2}\right\rangle=\int d^{3} \mathbf{r} \psi_{1}(\mathbf{r}) \frac{\hat{r}_{y}}{r_{\gamma i}^{3}} \frac{\partial}{\partial z} \psi_{2}(\mathbf{r})$, which exhibit a very sharp divergence at $\mathbf{r}_{i}=\mathbf{R}_{\gamma}$. A realspace implementation of these matrix elements is possible, however their numerical evaluation is very inefficient (at least in a plane wave code) because it requires a very large plane wave cutoff or, equivalently, a very small real-space grid, as shown in Fig. 3.

A more efficient solution is to evaluate this integral in the Fourier space of the electron orbitals and density. In fact, it is simple to prove that for any (non-singular) function $A(\mathbf{r})$ with Fourier transform $\tilde{A}(\mathbf{r})$ the following transformation holds: ${ }^{42}$

$$
\begin{aligned}
\boldsymbol{f}(\mathbf{R})= & \int \frac{\mathbf{r}-\mathbf{R}}{|\mathbf{r}-\mathbf{R}|^{3}} \times \boldsymbol{A}(\mathbf{r}) d^{3} \mathbf{r} \\
& \stackrel{F T}{\rightarrow} \tilde{\boldsymbol{f}}(\mathbf{G})=-4 \pi i \frac{\mathbf{G}}{G^{2}} \times \tilde{\boldsymbol{A}}(\mathbf{G}) .
\end{aligned}
$$

Finally, the quantity $\boldsymbol{f}(\mathbf{R})$ can be calculated at the position of the nucleus $\gamma$ using the relation $\boldsymbol{f}\left(\mathbf{R}=\mathbf{R}_{\gamma}\right)$ $=\sum_{\mathbf{G}} \tilde{\boldsymbol{f}}(\mathbf{G}) e^{-i \mathbf{G R}_{\gamma}}$

\section{APPLICATIONS: VALIDATION AND TESTS}

In order to validate our method and its implementation within the CPMD code, ${ }^{15}$ we have selected two small organic molecules, namely, formaldehyde and acetone for which we compute SOC matrix elements for the excited states ${ }^{1}\left(n, \pi^{*}\right)$, ${ }^{3}\left(n, \pi^{*}\right)$, and ${ }^{3}\left(\pi, \pi^{*}\right)$ (see Figs. 1 and 2 ). According to El-Sayed's rule ${ }^{43}$ any spin angular momentum change must
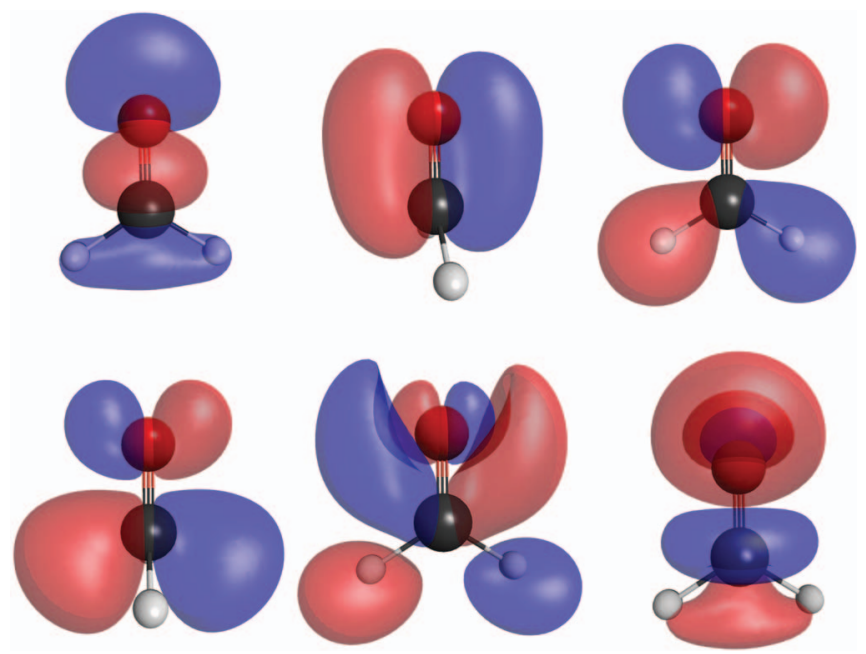

FIG. 1. Active space orbitals used in the CASSCF calculation for formaldehyde. From the right (top): HOMO-2, HOMO-1, HOMO; (bottom): LUMO, LUMO+1, LUMO+2. The corresponding DFT orbitals are undistinguishable from the CASSCF ones. 

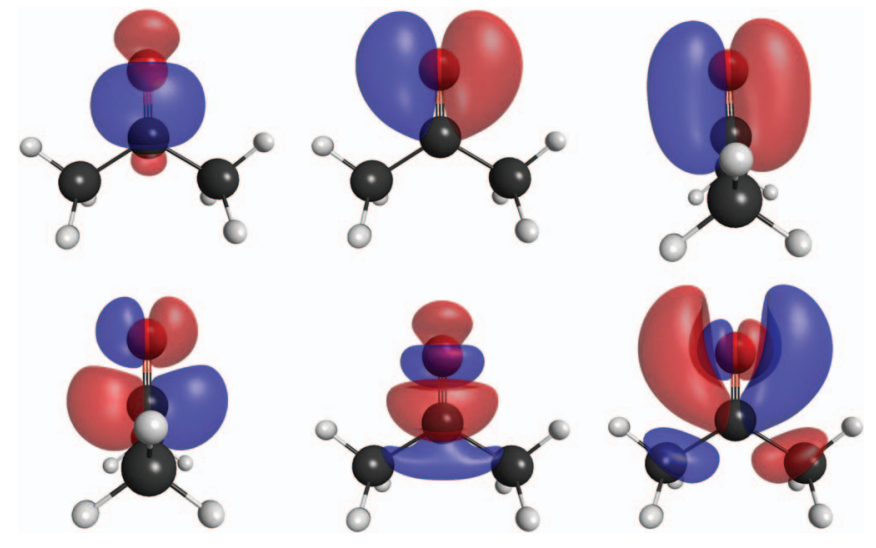

FIG. 2. Active space orbitals used in the CASSCF calculation for acetone. From the right (top): HOMO-2, HOMO-1, HOMO; (bottom): LUMO, $\mathrm{LUMO}+1$, LUMO+2. The corresponding DFT orbitals are undistinguishable from the CASSCF ones.

be accompanied by a change in the orbital angular momentum, so that total angular momentum is conserved. Consequently, by considering the coupling between these states we obtain one strong $\left({ }^{1}\left(n, \pi^{*}\right)\right.$ to $\left.{ }^{3}\left(\pi, \pi^{*}\right)\right)$ and one weak $\left({ }^{1}\left(n, \pi^{*}\right)\right.$ to $\left.{ }^{3}\left(n, \pi^{*}\right)\right)$ SOC matrix elements, proving a stringent validation of the approach. Our results obtained using Eqs. (17), (18), and (22) are compared with those computed with the quantum chemistry program GAMESS ${ }^{44}$ using the Spin-Orbit Multi-Configuration Quasi-Degenerate Perturbation Theory method for the one-electron SOC operator. ${ }^{45}$ This reference gives a high level of accuracy since it includes both static and dynamic correlation.

Table I summarizes the results obtained with our implementation using both a real-space (RS) and a reciprocal-space (GS) integration schemes for the matrix elements in Eq. (25), together with the GAMESS reference results.

For comparison, we also computed the two-electron contribution for formaldehyde at SO-GMCQDPT2 level of theory using GAMESS. ${ }^{44}$ We obtained a value of

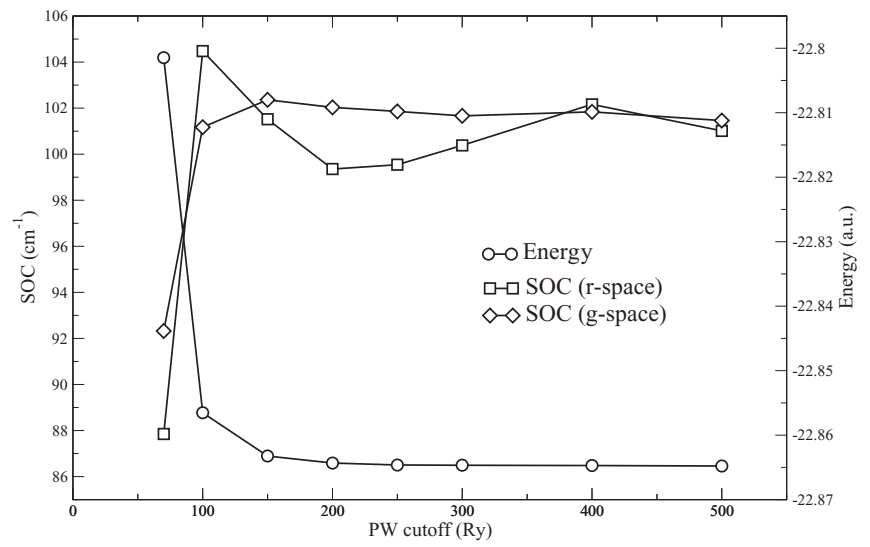

FIG. 3. Graph showing absolute value of $\left\langle{ }^{1}\left(n, \pi^{*}\right)\left|\hat{H}_{1 e l}^{S O}\right|{ }^{3}\left(\pi, \pi^{*}\right)_{0}\right\rangle$ for formaldehyde versus plane-wave cutoff in Rydberg. Squares and diamonds represent the results obtained using the real-space and g-space integrator, respectively. The total energy of the system (circles) is also plotted as a function of the cutoff value (right-hand side axis).

$-34.232 \mathrm{~cm}^{-1}$ for the two-electron term that corresponds to a full SOC of $56.853 \mathrm{~cm}^{-1}$.

For comparison, we also computed the full SOC using the ZORA Hamiltonian as implemented in the Amsterdam Density Functional program ${ }^{46}$ and obtained a value of $57.390 \mathrm{~cm}^{-1}$.

As discussed above, SOC integrals of the form given in Eq. (25) can be evaluated either in real space or in reciprocal space. However, due to divergencies at the positions of the nuclei, the calculation in real space requires a real space grid with an ultra-fine mesh, which corresponds to a very large plane wave cutoff value (>500 Ry). On the other hand, as shown in Fig. 3 the calculation of the SOCs converges smoothly in reciprocal space, and, for the systems studied in this work, a plane wave cutoff between 90 and 100 Ry is sufficient for achieving convergence; a value which is only slightly larger than the one required to converge the energy.

TABLE I. Absolute values of SOCs for formaldehyde and acetone computed for two pairs of weakly and strongly coupled states. All quantities are in units of $\mathrm{cm}^{-1}$. The total SOC coupling (last column) is given by the squared root of the sum of the squared components. The LR-TDDFT/PBE (see Sec. VI) are performed with the CPMD software using plane wave cutoff of $70 \mathrm{Ry}(\mathrm{l})$ and $300 \mathrm{Ry}(\mathrm{h}) .\left\langle^{1}(S)||^{3}(T)_{0}\right\rangle$ stands for $\left\langle{ }^{1}(S)\left|\hat{H}_{1 e l}^{S O}\right|^{3}(T)_{n}\right\rangle$, with $n \in\{-1,0,1\}$.

\begin{tabular}{|c|c|c|c|c|}
\hline Transitions & $\left|\left\langle{ }^{1}\left(n, \pi^{*}\right) \mid \|^{3}\left(\pi, \pi^{*}\right)_{0}\right\rangle\right|$ & $\left|\left\langle^{1}\left(n, \pi^{*}\right)||^{3}\left(\pi, \pi^{*}\right)+1\right\rangle\right|$ & $\mid\left\langle\left\langle^{1}\left(n, \pi^{*}\right)||^{3}\left(\pi, \pi^{*}\right)_{-1}\right\rangle\right|$ & Total \\
\hline \multicolumn{5}{|c|}{ Formaldehyde (strong coupling) } \\
\hline SO-GMCQDPT2 & 91.0845 & 0.0065 & 0.0065 & 91.0845 \\
\hline LR-TDDFT/PBE (1) & 92.9600 & 0.0001 & 0.0141 & 92.9737 \\
\hline LR-TDDFT/PBE (h) & 101.6651 & 0.0069 & 0.0069 & 101.6651 \\
\hline \multicolumn{5}{|c|}{ Acetone (strong coupling) } \\
\hline SO-GMCQDPT2 & 90.9543 & 0.0118 & 0.0118 & 90.9543 \\
\hline LR-TDDFT/PBE (1) & 85.4613 & 0.0047 & 0.0048 & 85.4189 \\
\hline LR-TDDFT/PBE (h) & 97.5095 & 0.0044 & 0.0044 & 97.5095 \\
\hline Transitions & $\left|\left\langle{ }^{1}\left(n, \pi^{*}\right) \|^{3}\left(n, \pi^{*}\right)_{0}\right\rangle\right|$ & $\left|\left\langle^{1}\left(n, \pi^{*}\right) \|^{3}\left(n, \pi^{*}\right)_{+1}\right\rangle\right|$ & $\mid\left\langle\left\langle^{1}\left(n, \pi^{*}\right)||^{3}\left(n, \pi^{*}\right)-1\right\rangle\right|$ & Total \\
\hline \multicolumn{5}{|c|}{ Formaldehyde (weak coupling) } \\
\hline SO-GMCQDPT2 & 0.0000 & 0.0001 & 0.0001 & 0.0001 \\
\hline LR-TDDFT/PBE (1) & 0.0011 & 0.0006 & 0.0022 & 0.0031 \\
\hline \multicolumn{5}{|c|}{ Acetone (weak coupling) } \\
\hline SO-GMCQDPT2 & 0.0000 & 0.0023 & 0.0023 & 0.0032 \\
\hline LR-TDDFT/PBE (1) & 0.0086 & 0.0001 & 0.0001 & 0.0086 \\
\hline
\end{tabular}




\section{A. Validation of the "spin-flip" calculations}

To test the accuracy of the SOC calculation for spinflip configurations using standard collinear LR-TDDFT, we have designed the following numerical experiment. For symmetry reasons, when the principal axis of the formaldehyde molecule is oriented along the $z$-axis, only the SOC matrix element between the states $\left|S_{I}^{0}\right\rangle$ and $\left|T_{J}^{0}\right\rangle$ is non-zero (also in the strong coupling case). This transition does not require a spinflip and therefore we expect our method to be in best agreement with the benchmark results. However, by performing a $90^{\circ}$ anticlockwise rotation of the Cartesian reference system around the $y$-axis (which corresponds to the mapping $x \rightarrow z^{\prime}$, $y \rightarrow y^{\prime}, z \rightarrow-x^{\prime}$ ), while keeping the molecule fixed in space (passive rotation), we achieved a rotation of the spin quantization axis from the $z$ to the $-x$ direction. Introducing the corresponding rotation matrix

$$
U_{y}^{\pi / 2}=\exp \left(-i \frac{\bar{\sigma} \cdot \hat{\boldsymbol{n}}(\pi / 2)}{2}\right)=\left(\begin{array}{cc}
\cos (\pi / 4) & \sin (\pi / 4) \\
-\sin (\pi / 4) & \cos (\pi / 4)
\end{array}\right),
$$

we get $\sigma_{y}^{\prime}=U_{y}^{\pi / 2} \cdot \sigma_{y} \cdot\left(U_{y}^{\pi / 2}\right)^{-1}$, where $\hat{\boldsymbol{n}}=(0,1,0)$ and $\bar{\sigma}=\left(\sigma_{1}, \sigma_{2}, \sigma_{3}\right)$.

The same SOC matrix elements should also arise from a numerical calculation in which it is the molecule that is rotated (in the opposite sense) while keeping the Cartesian axis still (active rotation). However, it is important to note that in this case the spin-flip contributions will result from the direct evaluation of the terms in Eqs. (19) and (20) and not from simple geometrical arguments as when the passive rotation was applied. From a comparison of the two results, the geometrical (passive rotation) and the numerical (active rotation), we can assess the quality of the spin-flip calculation using the standard collinear LR-TDDFT approach. The values reported in Table II show the surprisingly good agreement between the two approaches, also when compared with the reference SOGMCQDPT2 results.

We can therefore conclude that, at least for the case of simple molecular system, the collinear LR-TDDFT formalism provides very good results for SOC matrix elements between states with different $M_{S}$ values connected by spinflip transitions. However, if this agreement also holds in the case of more complex compounds, especially those containing heavier elements is not predictable and will require further investigations.
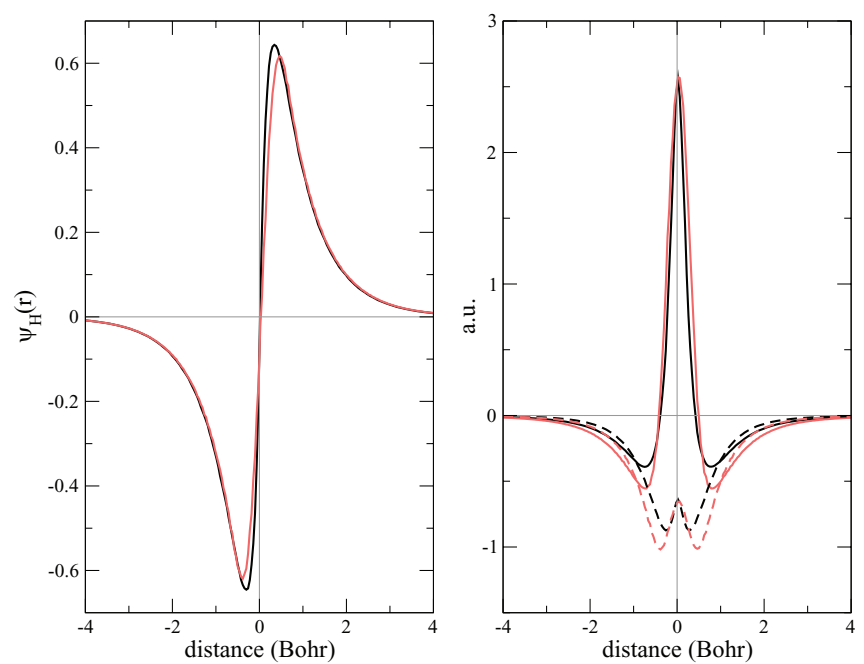

FIG. 4. Left: One-dimensional cut of the KS-HOMO orbital along a line passing through the Oxygen atom of formaldehyde and perpendicular to the $\mathrm{C}=\mathrm{O}$ bond. Right: One-dimensional cut along the same line for the quantities $f_{x y}$ (solid lines) and $f_{y x}$ (dashed lines) in Eq. (25) for $f_{x y}\left(\boldsymbol{r}^{\prime} ; \boldsymbol{R}_{O}\right)$ $=\psi_{H}^{*}\left(\mathbf{r}^{\prime}\right) \frac{\left(r_{x}^{\prime}-R_{x}\right) p_{y}}{\left|\mathbf{R}-\mathbf{r}^{\prime}\right|^{3}} \psi_{H-1}\left(\mathbf{r}^{\prime}\right)$. The curves obtained from PP-calculation at $100 \mathrm{Ry}$ (red) is in very good agreement with the all-electron calculations at 1800 Ry (black).

\section{B. Validation of the PP calculation}

In this section, we discuss the implications of the use of pseudopotentials (PP) for the calculation of SOCs. In electronic structure calculations with plane waves, it is a common practice to divide electrons into core (with large binding energy) and valence (with low-binding energy). The advantage of this separation is that the core electrons can be "frozen" in their atomic configuration while only valence and virtual orbitals are used to describe the chemical modifications, including electronic excitations. In this work, we make use of norm-conserving PP generated according to the Martins-Troullier scheme, ${ }^{47}$ with the possibility to include scalar-relativistic corrections. As shown in Fig. 4 for the case of the valence orbitals of formaldehyde, these types of PPs reproduce very accurately the KS orbitals of the valence electrons even in the region close to the nuclei. Therefore, when considering SOC matrix elements between singlet and triplet transitions involving valence KS orbitals (like in the case of the $\left(n, \pi^{*}\right)$ and $\left(\pi, \pi^{*}\right)$ transitions in formaldehyde and acetone) we obtained quite accurate values using the Breit-Pauli Hamiltonian of Eq. (6), without empirical

TABLE II. Absolute values of SOCs for formaldehyde computed for a pair of strongly coupled singlet to triplet transitions. All quantities are in units of $\mathrm{cm}^{-1}$ (Abbreviations are defined in Table I). The LR-TDDFT/PBE results obtained geometrically through a (passive) rotation of the Cartesian axes are labelled by "-GE," while the ones obtained using the approximated spin-flip approach are labelled with "-SF." ((l) and (h) have the same meaning as in Table I.)

\begin{tabular}{|c|c|c|c|c|}
\hline Transition & $\left|\left\langle^{1}\left(n, \pi^{*}\right) \|^{3}\left(\pi, \pi^{*}\right)_{0}\right\rangle\right|$ & $\left|\left\langle{ }^{1}\left(n, \pi^{*}\right)||^{3}\left(\pi, \pi^{*}\right)_{+1}\right\rangle\right|$ & $\left|\left\langle^{1}\left(n, \pi^{*}\right) \|^{3}\left(\pi, \pi^{*}\right)_{-1}\right\rangle\right|$ & Total \\
\hline \multicolumn{5}{|c|}{ Formaldehyde (strong coupling) } \\
\hline SO-GMCQDPT2 & 0.0000 & 64.4066 & 64.4066 & 91.0847 \\
\hline LR-TDDFT/PBE-SF (1) & 0.1188 & 65.5312 & 65.5312 & 92.6752 \\
\hline LR-TDDFT/PBE-SF (h) & 0.0113 & 71.8519 & 71.8519 & 101.6139 \\
\hline LR-TDDFT/PBE-GE (1) & 0.0057 & 65.5660 & 65.5660 & 92.7243 \\
\hline LR-TDDFT/PBE-GE (h) & 0.0111 & 72.1055 & 72.1055 & 101.9726 \\
\hline
\end{tabular}


reparameterization of the effective nuclear charges. ${ }^{48}$ Figure 4 shows cuts along a line perpendicular to the $\mathrm{C}=\mathrm{O}$ bond of formaldehyde and passing through the Oxygen atom for the KS-HOMO orbital (left-hand side) and the functions $f_{x y}\left(\boldsymbol{r}^{\prime} ; \boldsymbol{R}=\boldsymbol{R}_{O}\right)=\psi_{H}^{*}\left(\mathbf{r}^{\prime}\right) \frac{\left(r_{x}^{\prime}-R_{x}\right) p_{y}}{\left|\mathbf{R}-\mathbf{r}^{\prime}\right|^{3}} \psi_{H-1}\left(\mathbf{r}^{\prime}\right)$ and $f_{y x}\left(\boldsymbol{r}^{\prime} ; \boldsymbol{R}_{O}\right)$. The agreement between the all-electron (black lines) and the PP (red lines) is very good especially in the region of the atomic location (at distance equal to zero), at which position the SOC integrals are evaluated (left-hand side of Eq. (25)). The SOC interaction between core and valence electrons can also be taken into account using a sum of Coulomb and exchange-type operators. ${ }^{49}$

Finally, there may be situations in which SOC matrix elements involving pseudo-orbitals of the core become important. In this case, since their amplitude in the proximity of the nuclei are "artificially" small, the errors in the evaluation of the matrix elements of the Breit-Pauli Hamiltonian can become very large. To overcome these difficulties not only inplane wave but also in localized basis set calculations, several model spin-orbit coupling operators were developed. ${ }^{50-52} \mathrm{~A}$ discussion on these approximate schemes is however beyond the scope of this article.

\section{DISCUSSION AND CONCLUSIONS}

In this article, we have described a rigorous derivation of spin-orbit coupling matrix elements within LR-TDDFT. Our approach is based on the Breit-Pauli Hamiltonian as a perturbation to a standard, non-relativistic, LR-TDDFT calculation of the excitation energies and corresponding KS orbital transitions. The Tamm-Dancoff approximation was assumed throughout the derivation (and in the calculations) even if its extension to the full LR-TDDFT framework is straightforward. The SOC matrix elements are evaluated using the AMEWs introduced in Refs. 13 and 33, which have already been applied in the calculation of Tully's surface hopping couplings, and nonadiabatic coupling vectors. We presented an implementation of the numerical calculation of the SOCs in both Casida's ${ }^{16}$ and Sternheimer's ${ }^{19}$ formulation of LR-TDDFT within the framework of pseudopotentialbased calculations with a plane wave basis set. However, the same working equations can be also applied in the case of all-electron calculations using localized basis sets.

Our derivation is based on the collinear formulation of DFT and LR-TDDFT, which by construction does not allow for transitions involving spin-flip. To overcome this, a derivation of the SOC matrix elements in the noncollinear formalism of LR-TDDFT is required, but this is beyond the scope of the present work. However, we have shown that using the same linear response orbitals computed for a $S_{I}^{0} \rightarrow T_{J}^{0}$ transition it is possible to obtain accurate SOC values also for the "missing transitions" $\left(S_{I}^{0} \rightarrow T_{J}^{-1}\right.$ and $\left.S_{I}^{0} \rightarrow T_{J}^{1}\right)$, using collinear LR-TDDFT and standard (GGA) functionals and corresponding adiabatic TDDFT kernels. The quality of these calculations was assessed using geometrical arguments that allow us to obtain exact values for SOC matrix elements involving spin-flip transitions starting from those allowed in collinear LR-TDDFT. The agreement of our results, also in comparison with SO-GMCQDPT2 benchmark calculations, is in general very good, especially if we consider the fact that we are computing SOC of organic molecules which are of the order of $0.01 \mathrm{eV}$.

We have implemented the calculation of the SOC matrix elements in the pseudopotential plane wave code CPMD ${ }^{15}$ using the Sternheimer representation of LR-TDDFT. However, a full description of the implementation in the so-called Casida formalism is also presented and further discussed in Appendices B and C. We found out that a good accuracy is achieved using a reciprocal space integration of the $1 / r^{3}$ term of the Breit-Pauli Hamiltonian. In this case, we could obtain converged results for values of the plane wave cutoff that is just slightly larger than the one used in standard calculations using MT-type of PPs (80-90 Ry compared to 70 Ry for systems containing second row elements). We also noticed that, when the transitions taking part in the SOC calculation only involve (energetically) high-lying KS orbitals, the quality of their expansion in the PW basis set close to the position of the nuclei is very good, which reflects into accurate values of the corresponding SOCs. This was also confirmed by an all electron calculation with CPMD performed at a cutoff of 1800 Ry.

In conclusion, we have shown a rigorous derivation of the SOC matrix elements within the framework of LR-TDDFT, both in Casida's and Sternheimer's formulations. These calculations can be easily optimized for plane wave as well as localized basis sets and provide accurate SOC values with a negligible additional computational overhead compared to standard LR-TDDFT energy calculations. This can be applied to study a wide range of interesting phenomena, such as molecular magnetism, spin transport, and spin quantum dots dynamics for quantum computing. Regarding our particular interests, we now have the capability to extend nonadiabatic on-the-fly trajectory based approaches such as Tully's trajectory surface hopping (TSH) $)^{53-55}$ and nonadiabatic Bohmian Dynamics $^{56-58}$ to the dynamical study of intersystem crossing within LR-TDDFT, which are key to understanding many important photochemical and photophysical processes.

\section{COMPUTATIONAL DETAILS}

All the DFT/LR-TDDFT calculations were performed with the CPMD software package ${ }^{15}$ using Martins-Trouiller type pseudopontials ${ }^{47,59}$ and within the Tamm-Dancoff approximation. $^{60,61}$ The Perdew-Burke-Ernzerhof (PBE) exchange correlation functional ${ }^{62}$ was used in all DFT calculations. The value of the plane-wave energy cutoff is specified for each separate calculation. The box sizes used were $12 \times 8 \times 12$ a.u. for formaldehyde and $12 \times 12$ $\times 12$ a.u for acetone.

The benchmark wavefunction-theory calculations were performed with the quantum chemistry program GAMESS ${ }^{44}$ using a cc-pVDZ basis set. ${ }^{63}$ We began by performing a complete active space self-consistent field (CASSCF) calculation starting from orbitals obtained in a RHF calculation. The active space for the CASSCF was a (6/6) active space with three occupied orbitals and three virtual orbitals (see Figures 1 and 2). This active space was chosen to give an accurate 
description of the relevant electronic transitions of the carbonyl group (namely $n \rightarrow \pi^{*}, \pi \rightarrow \pi^{*}$, and $\sigma \rightarrow \pi^{*}$ ). We first performed a state-averaged CASSCF starting from the CASSCF results with all weights chosen to be equal (average over 5 states for acetone and 7 states for formaldehyde). In a second step all weights associated to the non relevant states: ${ }^{1}\left(\sigma, \pi^{*}\right),{ }^{3}\left(n, \pi^{*}\right)$, and the ground-state, are minimized. In the case of formaldehyde, the final weight of the ground state was set to zero and those of the $\left(\sigma, \pi^{*}\right)$ states became $8.8 \%$ of the value of all other relevant states. In the case of acetone the final weights for both the ground-state and the $\left(\sigma, \pi^{*}\right)$ states are set to zero. Subsequently, the state-averaged multiconfigurational wavenfunctions were used to perform a generalized multi-configurational quasi-degenerate second order perturbation theory calculation (G-MCQDPT2) ${ }^{64}$ in order to account for "dynamic electronic correlation." Finally, these results are used to obtain the values of the one-body spin-orbit coupling matrix elements of the Breit-Pauli Hamiltonian; the last two calculations were performed in a single run thanks to the GAMESS' SO-MCQDPT ${ }^{65}$ program, although SOC was not included in the perturbing Hamiltonian.

\section{ACKNOWLEDGMENTS}

The authors would like to acknowledge Dr. Francois P. Rotzinger for his help with the GAMESS' SO-MCQDPT calculations. COST actions CM0702 and CM1204, and the Swiss National Science Foundation (Grant Nos. 200021146396 and 200021-137717) are acknowledged for funding and support.

\section{APPENDIX A: THE BREIT-PAULI SO HAMILTONIAN}

The full Breit-Pauli SO Hamiltonian is composed by three terms

$$
\begin{aligned}
\hat{H}_{B P}^{S O}= & \frac{\alpha}{2}\left[\sum_{i}\left(-\nabla_{i}\left(\sum_{\gamma} \frac{Z_{\gamma}}{\hat{r}_{i \gamma}}\right) \times \hat{\mathbf{p}}_{i}\right) \cdot \hat{\mathbf{s}}_{i}\right. \\
& +\sum_{i} \sum_{j \neq i}\left(\nabla_{i}\left(\frac{1}{\hat{r}_{i j}}\right) \times \hat{\mathbf{p}}_{i}\right) \cdot \hat{\mathbf{s}}_{i} \\
& \left.+2 \sum_{i} \sum_{j \neq i}\left(\nabla_{j}\left(\frac{1}{\hat{r}_{i j}}\right) \times \hat{\mathbf{p}}_{j}\right) \cdot \hat{\mathbf{s}}_{i}\right] .
\end{aligned}
$$

The first term is the one-electron spin-same-orbit term (same index for the $\hat{\mathbf{p}}_{i}$ and $\hat{\mathbf{s}}_{i}$ operators). The other two are two-electron contributions (based on the Coulomb repulsion between a pair of electrons in position $\mathbf{r}_{i}$ and $\mathbf{r}_{j}$ ): a spin-sameorbit term and a spin-other-orbit term.

\section{APPENDIX B: AMEW FOR SINGLET AND TRIPLET STATES}

Consider Casida's equation $\Omega \mathbf{F}_{I}=\omega_{I} \mathbf{F}_{I}$. We can write the matrix $\Omega$ in block-matrix form by arranging its elements according to their spin index,

$$
\left(\begin{array}{ll}
\Omega_{\alpha \alpha} & \Omega_{\alpha \beta} \\
\Omega_{\beta \alpha} & \Omega_{\beta \beta}
\end{array}\right)
$$

and in the case of a closed shell molecule this matrix becomes even simpler because the ground state density is not spin-polarized, thus $\Omega_{\alpha \beta}=\Omega_{\beta \alpha}$ and $\Omega_{\alpha \alpha}=\Omega_{\beta \beta}$.

Starting from

$$
\Omega=\left(\begin{array}{cc}
\Omega_{\alpha \alpha} & \Omega_{\alpha \beta} \\
\Omega_{\alpha \beta} & \Omega_{\alpha \alpha}
\end{array}\right)
$$

and applying the unitary transformation $U=\left(\begin{array}{cc}\mathbb{1} & \mathbb{1} \\ \mathbb{1} & -\mathbb{1}\end{array}\right)$,

$$
U^{\dagger} \Omega U=\left(\begin{array}{cc}
\Omega_{\alpha \alpha}+\Omega_{\alpha \beta} & 0 \\
0 & \Omega_{\alpha \alpha}-\Omega_{\alpha \beta}
\end{array}\right)
$$

we get a block diagonal matrix, and therefore we can solve the sub-problems separately,

$$
\left(\Omega_{\alpha \alpha}+\Omega_{\alpha \beta}\right) \mathbf{f}^{S}=\omega_{S}^{2} \mathbf{f}^{S}
$$

and

$$
\left(\Omega_{\alpha \alpha}-\Omega_{\alpha \beta}\right) \mathbf{f}^{T}=\omega_{T}^{2} \mathbf{f}^{T},
$$

and use their solutions to find the eigenvectors of $U^{\dagger} \Omega U$, which are

$$
\mathbf{F}^{S}=\left(\begin{array}{c}
\mathbf{f}^{S} \\
0
\end{array}\right) \quad \text { and } \quad \mathbf{F}^{T}=\left(\begin{array}{c}
0 \\
\mathbf{f}^{T}
\end{array}\right) .
$$

Using $U$ to get the solutions the original problem, we finally get

$$
U \mathbf{F}^{S}=\left(\begin{array}{c}
\mathbf{f}^{S} \\
\mathbf{f}^{S}
\end{array}\right) \text { and } U \mathbf{F}^{T}=\left(\begin{array}{c}
\mathbf{f}^{T} \\
-\mathbf{f}^{T}
\end{array}\right)
$$

which shows how the solutions of Casida's equations indeed display the pattern that makes the AMEW be either a singlet or a triplet. The same result also holds within the Sternheimer formulation of LR-TDDFT.

\section{APPENDIX C: EVALUATING SOC MATRIX ELEMENTS WITH AMEWs}

From Eq. (22), we observe that for each matrix element evaluated for SD we will need to compute $N \times N$ one-electron matrix elements. If we consider a four-electron system with ground state wavefunction $\left|\Psi_{0}\right\rangle=\left|\begin{array}{llll}1 & \overline{1} & 2 & \overline{2}\end{array}\right\rangle$, this means a total $4 \times 4 \times 16$ one-electron matrix elements for $\left\langle S_{1}^{0}\left|\hat{H}_{1 e l}^{S O}\right| T_{1}^{0}\right\rangle$ and $4 \times 4 \times 8$ for $\left\langle S_{1}^{0}\left|\hat{H}_{1 e l}^{S O}\right| T_{1}^{-1,+1}\right\rangle$. Fortunately, since the linear-response orbitals are orthogonal to the ground state KS orbitals, it is possible to simplify this task by deriving new rules to compute these matrix elements. Let us first look at 
$\left\langle S_{1}^{0}\left|\hat{H}_{1 e l}^{S O}\right| T_{1}^{0}\right\rangle$. In our four-electron system, we have

$$
\begin{aligned}
& \left\langle\psi_{S}\left|\hat{H}^{S O}\right| \psi_{T}\right\rangle \\
& =\left\langle\phi_{1}^{S} \overline{1} 2 \overline{2}\left|\hat{H}^{S O}\right| \phi_{1}^{T} \overline{1} 2 \overline{2}\right\rangle+\left\langle\phi_{1}^{S} \overline{1} 2 \overline{2}\left|\hat{H}^{S O}\right| \begin{array}{llll}
1 & \bar{\phi}_{1}^{T} & 2 & \overline{2}
\end{array}\right. \\
& +\left\langle\phi_{1}^{S} \overline{1} 2 \overline{2}\left|\hat{H}^{S O}\right| 1 \quad \overline{1} \phi_{2}^{T} \overline{2}\right\rangle+\left\langle\phi_{1}^{S} \overline{1} 2 \overline{2}\left|\hat{H}^{S O}\right| 1 \overline{1} 2 \bar{\phi}_{2}^{T}\right\rangle
\end{aligned}
$$

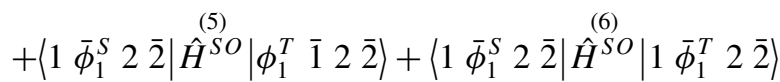

$$
\begin{aligned}
& +\left\langle 1 \bar{\phi}_{1}^{S} 2 \overline{2}\left|\hat{H}^{(7)}\right| 1 \overline{1} \phi_{2}^{T} \overline{2}\right\rangle+\left\langle 1 \bar{\phi}_{1}^{S} 2 \overline{2}^{(8)}\left|\hat{H}^{S O}\right| 1 \overline{1} 2 \bar{\phi}_{2}^{T}\right\rangle
\end{aligned}
$$

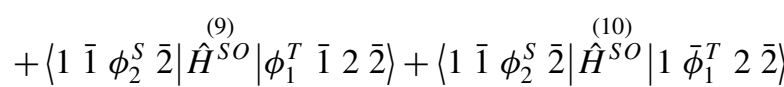

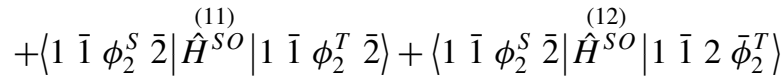

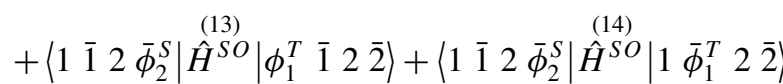

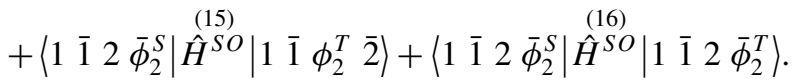

Only eight out of these sixteen matrix elements will be nonzero and we are able to distinguish two cases in which this happens. The first one is when the two SDs in the matrix element are created by the same excitation. This happens with matrix elements (1), (6), (11), and (16) in Eq. (C1). Let us see what happens when we apply Eq. (22) to matrix elements (1). The overlap matrix (Eq. (23)) will be

$$
S_{S T}=\left(\begin{array}{cccc}
\left\langle\phi_{1}^{S} \mid \phi_{1}^{T}\right\rangle & 0 & 0 & 0 \\
0 & 1 & 0 & 0 \\
0 & 0 & 1 & 0 \\
0 & 0 & 0 & 1
\end{array}\right) .
$$

Notice that only four of the possible cofactors will give nonzero contributions, namely, those defined by the elements along the diagonal. These will give

$$
\begin{gathered}
(-1)^{2+2}\left|\begin{array}{cccc}
\left\langle\phi_{1}^{S} \mid \phi_{1}^{T}\right\rangle & \square & 0 & 0 \\
\square & \square & \square & \square \\
0 & \square & 1 & 0 \\
0 & \square & 0 & 1
\end{array}\right|=\left|\begin{array}{ccc}
\left\langle\phi_{1}^{S} \mid \phi_{1}^{T}\right\rangle & 0 & 0 \\
0 & 1 & 0 \\
0 & 0 & 1
\end{array}\right| \\
=\left\langle\phi_{1}^{S} \mid \phi_{1}^{T}\right\rangle
\end{gathered}
$$

or

$$
(-1)^{1+1}\left|\begin{array}{cccc}
\square & \square & \square & \square \\
\square & 1 & 0 & 0 \\
\square & 0 & 1 & 0 \\
\square & 0 & 0 & 1
\end{array}\right|=\left|\begin{array}{lll}
1 & 0 & 0 \\
0 & 1 & 0 \\
0 & 0 & 1
\end{array}\right|=1,
$$

and therefore, using the rule given in Eq. (22), the matrix element (1) will become

$$
\begin{aligned}
(1)= & \left\langle\phi_{1}^{S}\left|\hat{h}^{S O}\right| \phi_{1}^{T}\right\rangle+\left\langle\overline{1}\left|\hat{h}^{S O}\right| \overline{1}\right\rangle\left\langle\phi_{1}^{S} \mid \phi_{1}^{T}\right\rangle \\
& +\left\langle 2\left|\hat{h}^{S O}\right| 2\right\rangle\left\langle\phi_{1}^{S} \mid \phi_{1}^{T}\right\rangle+\left\langle\overline{2}\left|\hat{h}^{S O}\right| \overline{2}\right\rangle\left\langle\phi_{1}^{S} \mid \phi_{1}^{T}\right\rangle .
\end{aligned}
$$

The square symbols represent the matrix elements of the rows and columns that are stricken out in the calculation of the determinants. The other case in which a matrix element between
SDs will be non-zero is when the determinants are created by different excitations (excitations from different occupied KS orbitals), and the linear-response orbitals have the same spin. This happens with matrix elements (3), (8), (9), and (14) in Eq. (C1). Let us take a closer look at how to apply Eq. (22) to the matrix element (3). First of all, the overlap matrix becomes

$$
S_{S T}=\left(\begin{array}{cccc}
0 & 0 & \left\langle\phi_{1}^{S} \mid \phi_{2}^{T}\right\rangle & 0 \\
0 & 1 & 0 & 0 \\
0 & 0 & 0 & 0 \\
0 & 0 & 0 & 1
\end{array}\right),
$$

from which we can clearly see that only one cofactor will be non-zero, namely

$$
\begin{gathered}
(-1)^{(1+3)}\left(\begin{array}{cccc}
\square & 0 & \left\langle\phi_{1}^{S} \mid \phi_{2}^{T}\right\rangle & 0 \\
\square & 1 & 0 & 0 \\
\square & \square & \square & \square \\
\square & 0 & 0 & 1
\end{array}\right)=\left|\begin{array}{ccc}
0 & \left\langle\phi_{1}^{S} \mid \phi_{2}^{T}\right\rangle & 0 \\
1 & 0 & 0 \\
0 & 0 & 1
\end{array}\right| \\
=-\left|\begin{array}{ccc}
\left\langle\phi_{1}^{S} \mid \phi_{2}^{T}\right\rangle & 0 & 0 \\
0 & 1 & 0 \\
0 & 0 & 1
\end{array}\right|=-\left\langle\phi_{1}^{S} \mid \phi_{2}^{T}\right\rangle,
\end{gathered}
$$

from which we finally get

$$
\text { (3) }=-\left\langle 1\left|\hat{h}^{S O}\right| 2\right\rangle\left\langle\phi_{2}^{T} \mid \phi_{2}^{T}\right\rangle \text {. }
$$

Matrix elements (2), (4), (5), (7), (10), (12), (13), and (15) will all be zero.

Concerning the triplets $\left|T_{1}^{+1}\right\rangle$ and $\left|T_{1}^{-1}\right\rangle$, only eight matrix elements will have to be computed. As an example, we compute

$$
\begin{aligned}
& \left\langle S_{0}^{0}\left|\hat{H}^{S O}\right| T^{-1}\right\rangle \\
& =\left\langle\phi_{1}^{S} \overline{1} 2 \overline{2}\left|\hat{H}^{S O}\right| \overline{1} \bar{\phi}_{1}^{T} 2 \overline{2}\right\rangle+\left\langle\phi_{1}^{S} \overline{1} 2 \overline{2}\left|\hat{H}^{S O}\right| 1 \overline{1} \overline{2} \bar{\phi}_{2}^{T}\right\rangle \\
& \quad+\left\langle 1 \bar{\phi}_{1}^{S} 2 \overline{2}\left|\hat{H}^{S O}\right| \overline{1} \bar{\phi}_{1}^{T} 2 \overline{2}\right\rangle+\left\langle 1 \bar{\phi}_{1}^{S} 2 \overline{2}\left|\hat{H}^{(4)}\right| 1 \overline{1} \overline{2} \bar{\phi}_{2}^{T}\right\rangle \\
& \quad+\left\langle 1 \overline{1} \phi_{2}^{S} \overline{2}\left|\hat{H}^{S O}\right| \overline{1} \bar{\phi}_{1}^{T} 2 \overline{2}\right\rangle+\left\langle 1 \overline{1} \phi_{2}^{S} \overline{2}\left|\hat{H}^{S O}\right| 1 \overline{1} \overline{2} \bar{\phi}_{2}^{T}\right\rangle \\
& \quad+\left\langle 1 \overline{1} 2 \bar{\phi}_{2}^{S}\left|\hat{H}^{(7)}\right| \overline{1}_{1}^{S} \bar{\phi}_{1}^{T} 2 \overline{2}\right\rangle+\left\langle 1 \overline{1} 2 \bar{\phi}_{2}^{S}\left|\hat{H}^{(8)}\right| 1 \overline{1} \overline{2} \bar{\phi}_{2}^{T}\right\rangle .
\end{aligned}
$$

Thus, there will be three cases in which the matrix elements are non vanishing. The first will occur when the two SDs are created by the same excitation but have different spins, as is the case of matrix element (1), for which we have

$$
S_{S T=}\left(\begin{array}{cccc}
0 & 0 & 0 & 0 \\
0 & 1 & 0 & 0 \\
0 & 0 & 1 & 0 \\
0 & 0 & 0 & 1
\end{array}\right) .
$$

This matrix has a row and a column of zeros. Therefore, the only non-zero cofactor that can be generated is the one obtained by striking out the first line and the first column

$$
(-1)^{1+1}\left|\begin{array}{cccc}
\square & \square & \square & \square \\
\square & 1 & 0 & 0 \\
\square & 0 & 1 & 0 \\
\square & 0 & 0 & 1
\end{array}\right|=\left|\begin{array}{lll}
1 & 0 & 0 \\
0 & 1 & 0 \\
0 & 0 & 1
\end{array}\right|=1,
$$


which leads to the result

$$
\text { (1) }=\left\langle\phi_{1}^{S}\left|\hat{h}^{S O}\right| \bar{\phi}_{1}^{T}\right\rangle \text {. }
$$

This case will also apply to matrix element (6),

$$
(6)=(-1)^{3+3}\left|\begin{array}{ccc}
1 & 0 & 0 \\
0 & 1 & 0 \\
0 & 0 & 1
\end{array}\right|\left\langle\phi_{2}^{S}\left|\hat{h}^{S O}\right| \bar{\phi}_{2}{ }^{T}\right\rangle=\left\langle\phi_{2}^{S}\left|\hat{h}^{S O}\right| \bar{\phi}_{2}{ }^{T}\right\rangle \text {. }
$$

For the matrix elements (3) and (8) we have linear response orbitals that have been created by the same excitation and have the same spin. Therefore,

$$
\begin{aligned}
\text { (3) } & =\left\langle 1\left|\hat{h}^{S O}\right| \overline{1}\right\rangle(-1)^{1+2}\left|\begin{array}{ccc}
\left\langle\phi_{1}^{S} \mid \phi_{1}^{T}\right\rangle & 0 & 0 \\
0 & 1 & 0 \\
0 & 0 & 1
\end{array}\right| \\
& =-\left\langle 1\left|\hat{h}^{S O}\right| \overline{1}\right\rangle\left\langle\phi_{1}^{S} \mid \phi_{1}^{T}\right\rangle
\end{aligned}
$$

and

$$
\begin{aligned}
(8) & =\left\langle 1\left|\hat{h}^{S O}\right| \overline{1}\right\rangle(-1)^{1+2}\left|\begin{array}{ccc}
\left\langle\phi_{1}^{S} \mid \phi_{1}^{T}\right\rangle & 0 & 0 \\
0 & 1 & 0 \\
0 & 0 & 1
\end{array}\right| \\
& =-\left\langle 1\left|\hat{h}^{S O}\right| \overline{1}\right\rangle\left\langle\phi_{1}^{S} \mid \phi_{1}^{T}\right\rangle .
\end{aligned}
$$

Finally, when the SDs are created by different excitations but the linear-response orbitals have the same spin, as in the cases of (4) and (7), we have

$$
\begin{aligned}
\text { (4) } & =\left\langle 1\left|\hat{h}^{S O}\right| \overline{1}\right\rangle(-1)^{1+2}\left|\begin{array}{ccc}
\left\langle\phi_{1}^{S} \mid \phi_{1}^{T}\right\rangle & 0 & 0 \\
0 & 1 & 0 \\
0 & 0 & 1
\end{array}\right| \\
& =-\left\langle 1\left|\hat{h}^{S O}\right| \overline{1}\right\rangle\left\langle\phi_{1}^{S} \mid \phi_{1}^{T}\right\rangle
\end{aligned}
$$

and

$$
\begin{aligned}
\text { (7) } & =\left\langle 1\left|\hat{h}^{S O}\right| \overline{1}\right\rangle(-1)^{1+2}\left|\begin{array}{ccc}
\left\langle\phi_{1}^{S} \mid \phi_{1}^{T}\right\rangle & 0 & 0 \\
0 & 1 & 0 \\
0 & 0 & 1
\end{array}\right| \\
& =-\left\langle 1\left|\hat{h}^{S O}\right| \overline{1}\right\rangle\left\langle\phi_{1}^{S} \mid \phi_{1}^{T}\right\rangle .
\end{aligned}
$$

Noticing that the matrix elements (2) and (5) are zero, we get to the final result,

$$
\begin{aligned}
\left\langle S_{0}^{0}\right| & \hat{H}^{S O}\left|T^{-1}\right\rangle \\
= & -\left\langle 1\left|\hat{h}^{S O}\right| \overline{1}\right\rangle\left\langle\phi_{1}^{S} \mid \phi_{1}^{T}\right\rangle-\left\langle 2\left|\hat{h}^{S O}\right| \overline{2}\right\rangle\left\langle\phi_{2}^{S} \mid \phi_{2}^{T}\right\rangle \\
& -\left\langle 1\left|\hat{h}^{S O}\right| \overline{2}\right\rangle\left\langle\phi_{2}^{S} \mid \phi_{1}^{T}\right\rangle-\left\langle 2\left|\hat{h}^{S O}\right| \overline{1}\right\rangle\left\langle\phi_{1}^{S} \mid \phi_{2}^{T}\right\rangle \\
& +\left\langle\phi_{1}^{S}\left|\hat{h}^{S O}\right| \bar{\phi}_{1}^{T}\right\rangle+\left\langle\phi_{2}^{S}\left|\hat{h}^{S O}\right| \bar{\phi}_{2}^{T}\right\rangle \\
& +0+0 .
\end{aligned}
$$

The same type of analysis can be applied to $\left\langle S_{0}^{0}\left|\hat{H}^{S O}\right| T^{+1}\right\rangle$, to give

$$
\begin{aligned}
\left\langle S_{0}^{0}\right| & \hat{H}^{S O}\left|T^{+1}\right\rangle \\
= & -\left\langle\overline{1}\left|\hat{h}^{S O}\right| 1\right\rangle\left\langle\phi_{1}^{S} \mid \phi_{1}^{T}\right\rangle-\left\langle\overline{2}\left|\hat{h}^{S O}\right| 2\right\rangle\left\langle\phi_{2}^{S} \mid \phi_{2}^{T}\right\rangle \\
& -\left\langle\overline{1}\left|\hat{h}^{S O}\right| 2\right\rangle\left\langle\phi_{2}^{S} \mid \phi_{1}^{T}\right\rangle-\left\langle\overline{2}\left|\hat{h}^{S O}\right| 1\right\rangle\left\langle\phi_{1}^{S} \mid \phi_{2}^{T}\right\rangle \\
& +\left\langle\overline{\phi_{1}^{S}}\left|\hat{h}^{S O}\right| \phi_{1}^{T}\right\rangle+\left\langle\bar{\phi}_{2}^{S}\left|\hat{h}^{S O}\right| \phi_{2}^{T}\right\rangle \\
& +0+0 .
\end{aligned}
$$

${ }^{1}$ T. Saue, ChemPhysChem 12, 3077 (2011).

${ }^{2}$ P. Pyykkö, Annu. Rev. Phys. Chem. 63, 45 (2012).

${ }^{3}$ J. Autschbach, J. Chem. Phys. 136, 150902 (2012).

${ }^{4}$ G. Tarczay, A. G. Császár, W. Klopper, and H. M. Quiney, Mol. Phys. 99, 1769 (2001).

${ }^{5}$ W. Kohn and L. J. Sham, Phys. Rev. 140, A1133 (1965).

${ }^{6}$ E. Runge and E. K. U. Gross, Phys. Rev. Lett. 52, 997 (1984).

${ }^{7}$ A. Rajagopal, J. Phys. C 11, L943 (1978).

${ }^{8}$ A. MacDonald and S. H. Vosko, J. Phys. C 12, 2997 (1979).

${ }^{9}$ F. Wang, T. Ziegler, E. van Lenthe, S. van Gisbergen, and E. J. Baerends, J. Chem. Phys. 122, 204103 (2005).

${ }^{10}$ F. Wang and T. Ziegler, J. Chem. Phys. 123, 154102 (2005).

${ }^{11}$ I. Tavernelli, E. Tapavicza, and U. Rothlisberger, J. Chem. Phys. 130, 124107 (2009).

${ }^{12}$ I. Tavernelli, E. Tapavicza, and U. Rothlisberger, J. Mol. Struct.: THEOCHEM 914, 22 (2009).

${ }^{13}$ I. Tavernelli, B. F. E. Curchod, and U. Rothlisberger, J. Chem. Phys. 131, 196101 (2009).

${ }^{14}$ I. Tavernelli, B. F. E. Curchod, A. Laktionov, and U. Rothlisberger, J. Chem. Phys. 133, 194104 (2010).

${ }^{15}$ CPMD, copyright IBM Corp 1990-2008, Copyright MPI für Festkörperforschung Stuttgart 1997-2001, see http://www.cpmd.org/.

${ }^{16}$ M. E. Casida in Recent Advances in Density Functional Methods, edited by D. P. Chong (World Scientific, Singapore, 1995), p. 155.

${ }^{17}$ S. G. Chiodo and N. Russo, Chem. Phys. Lett. 490, 90 (2010).

${ }^{18}$ Q. Ou and J. E. Subotnik, J. Phys. Chem. C 117, 19839 (2013).

${ }^{19}$ R. Sternheimer, Phys. Rev. 84, 244 (1951).

${ }^{20}$ J. Hutter, J. Chem. Phys. 118, 3928 (2003).

${ }^{21}$ G. Granucci, M. Persico, and G. Spighi, J. Chem. Phys. 137, 22A501 (2012).

${ }^{22}$ P. Marquetand, M. Richter, J. González-Vázquez, I. Sola, and L. González, Faraday Discuss. 153, 261 (2011).

${ }^{23}$ A. K. Rajagopal and J. Callaway, Phys. Rev. B 7, 1912 (1973).

${ }^{24}$ R. M. Dreizler and E. K. U. Gross, Density Functional Theory (Springer, Berlin, 1990).

${ }^{25}$ U. von Barth and L. Hedin, J. Phys. C: Solid State Phys. 5, 1629 (1972).

${ }^{26}$ O. Gunnarsson and B. I. Lundqvist, Phys. Rev. B 13, 4274 (1976).

${ }^{27}$ C. A. Ullrich, Time-Dependent Density-Functional Theory (Oxford University Press, 2012).

${ }^{28}$ K. L. Liu and S. H. Vosko, Can. J. Phys. 67, 1015 (1989).

${ }^{29}$ Y. Shao, M. Head-Gordon, and A. I. Krylov, J. Chem. Phys. 118, 4807 (2003).

${ }^{30}$ F. Wang and T. Ziegler, J. Chem. Phys. 121, 12191 (2004).

${ }^{31}$ F. Wang and T. Ziegler, J. Chem. Phys. 122, 074109 (2005).

${ }^{32}$ Time-Dependent Density Functional Theory, Lecture Notes in Physics, edited by M. A. L. Marques, C. A. Ullrich, F. Nogueira, A. Rubio, K. Burke, and E. K. U. Gross (Springer, Berlin, 2006).

${ }^{33}$ E. Tapavicza, I. Tavernelli, and U. Rothlisberger, Phys. Rev. Lett. 98, 023001 (2007)

${ }^{34}$ M. A. L. Marques and E. K. U. Gross, Lect. Notes Phys. 620, 144 (2003).

${ }^{35}$ The definition of the TDDFT kernel $K(\omega)$ already incorporates the adiabatic approximation, in which $\delta v_{x c}^{\sigma}(\mathbf{r}, t) / \delta \rho_{\tau}\left(\mathbf{r}^{\prime}, t^{\prime}\right)=\delta\left(t-t^{\prime}\right) \delta^{2} E_{x c} /$ $\left(\delta \rho_{\sigma}(\mathbf{r}, t) \delta \rho_{\tau}\left(\mathbf{r}^{\prime}, t^{\prime}\right)\right)$, where $v_{x c}$ is the exchange-correlation potential.

${ }^{36}$ X. Gonze and J.-P. Vigneron, Phys. Rev. B 39, 13120 (1989).

${ }^{37}$ X. Gonze, Phys. Rev. A 52, 1096 (1995).

${ }^{38}$ X. Andrade, S. Botti, M. A. L. Marques, and A. Rubio, J. Chem. Phys. 126, 184106 (2007).

${ }^{39} \mathrm{R}$. McWeeny, Methods of Molecular Quantum Mechanics (Academic Press, 1992).

${ }^{40}$ P.-O. Löwdin, Phys. Rev. 97, 1474 (1955).

${ }^{41}$ B. A. Hess, C. M. Marian, U. Wahgren, and O. Groppen, Chem. Phys. Lett. 251, 365 (1996).

${ }^{42}$ D. Sebastiani and M. Parrinello, J. Chem. Phys. 105, 1951 (2001).

${ }^{43}$ M. A. El-Sayed, J. Chem. Phys. 38, 2834 (1963).

${ }^{44}$ M. W. Schmidt, K. K. Baldridge, J. A. Boatz, S. T. Elber, M. S. Gordon, J. H. Jensen, S. Koseki, N. Matsunaga, K. A. Nguyen, S. J. Su et al., J. Comput. Chem. 14, 1347-1363 (1993).

${ }^{45}$ Perturbation theory calculation based on a wavefunction obtained from a complete-active space multi-configuration self-consistent field calculation (CASSCF)

${ }^{46}$ ADF2009.01, SCM, Theoretical Chemistry, Vrije Universiteit, Amsterdam, The Netherlands, see http://www.scm.com, 2009.

${ }^{47}$ N. Troullier and J. L. Martins, Phys. Rev. B 43, 8861 (1991). 
${ }^{48}$ S. Koseki, M. S. Gordon, M. W. Schmidt, and N. Matsunga, J. Phys. Chem. 99, 12764 (1995).

${ }^{49}$ C. M. Marian, "Berechnung von Matrixelementen des Spin-Bhan- und Spin-Spin-Kopplungsoperators mit MRD-CI-Wellenfunktionen," Ph.D. thesis (University of Bonn, 1981).

${ }^{50}$ Y. S. Lee, W. C. Ermler, and K. S. Pitzer, J. Phys. Chem. 67, 5861 (1977)

${ }^{51}$ P. Hafner and W. H. Schwarz, J. Phys. B 11, 217 (1978).

${ }^{52}$ C. H. Teichteil, M. PÍissier, and F. Speigelmann, Chem. Phys. 81, 273 (1983).

${ }^{53}$ J. C. Tully and R. K. Preston, J. Chem. Phys. 55, 562 (1971).

${ }^{54}$ L. Martínez-Fernández, I. Corral, G. Granucci, and M. Persico, Chem. Sci. 5, 1336 (2014).

${ }^{55}$ M. Richter, P. Marquetand, J. González-Vázquez, I. Sola, and L. González, J. Chem. Theory Comput. 7, 1253 (2011).
${ }^{56}$ B. F. E. Curchod, I. Tavernelli, and U. Rothlisberger, Phys. Chem. Chem. Phys. 13, 3231 (2011).

${ }^{57}$ B. F. E. Curchod and I. Tavernelli, J. Chem. Phys. 138, 184112 (2013).

${ }^{58}$ B. F. E. Curchod, U. Rothlisberger, and I. Tavernelli, ChemPhysChem 14, 1314 (2013).

${ }^{59}$ N. Troullier and J. L. Martins, Phys. Rev. B 43, 1993 (1991).

${ }^{60}$ I. Tamm, J. Phys. (Moscow) 9, 449 (1945).

${ }^{61}$ S. Dancoff, Phys. Rev. 78, 382 (1950).

${ }^{62}$ J. P. Perdew, K. Burke, and M. Ernzerhof, Phys. Rev. Lett. 77, 3865 (1996).

${ }^{63}$ T. H. J. Dunning, J. Chem. Phys. 90, 1007 (1989).

${ }^{64}$ H. Nakano, J. Chem. Phys. 99, 7983 (1993).

${ }^{65}$ D. G. Fedorov and J. P. Finley, Phys. Rev. A 64, 042502 (2001). 